\title{
Endoscopic sinus surgery: evolution and technical innovations
}

\author{
S GovindaraJ, N D AdAPPA, D W KenNedy*
}

\begin{abstract}
Prior to the introduction of functional endoscopic sinus surgery, several surgeons had begun to use telescopes to perform surgical procedures in the nose and sinuses. However, the central concepts of functional endoscopic sinus surgery evolved primarily from Messerklinger's endoscopic study of mucociliary clearance and endoscopic detailing of intranasal pathology. The popularity of a combination of endoscopic ethmoidectomy plus opening of secondarily involved sinuses grew rapidly during the latter part of the twentieth century, and endoscopic intranasal techniques began to expand to deal with pathology other than inflammation. We present a review of the evolution of knowledge regarding the pathogenesis of inflammatory sinus disease since that point in time, and of the impact that this has had on the management of inflammatory sinus disease. We also detail the technological advances that have allowed endoscopic intranasal techniques to expand and successfully treat other pathology, including skull base and orbital disease. In addition, we describe evolving technologies which may further influence development within this field.
\end{abstract}

Key words: Endoscopy; Paranasal Sinus; Technology; Evolution

\section{Introduction}

In the 1970s, reports of endoscopic endonasal and intrasinus surgical interventions were produced by Wigand, Draf and other endoscopic intranasal pioneers. However, the central concepts of functional endoscopic sinus surgery (FESS) evolved primarily out of the detailed work of Messerklinger in evaluating mucociliary clearance patterns and endoscopic changes within the ostiomeatal complex and in incorporating computed tomography (CT) to image the ethmoid sinuses. ${ }^{1}$ The principles of FESS were first published in 1985, and the first two courses on endoscopic intranasal surgery were held at the Johns Hopkins Hospital in the same year.,3 Following this, there was rapid growth of both interest in and utilisation of endoscopic intranasal surgery.

Prior to the introduction of FESS, the majority of ethmoid procedures were done via external incisions or with a headlight intranasally, but surgery was more frequently aimed at the maxillary or even the frontal sinuses. ${ }^{4}$ The operating microscope was introduced to aid intranasal ethmoidectomy; however, although visualisation was enhanced, its use did not gain widespread acceptance. Despite providing a magnified image, the operating microscope had a number of limitations. The ability to obtain a binocular view through the nasal aperture proved to be inconsistent, and a direct line of sight was necessary. The subsequent introduction of the concepts of endoscopic surgical intervention revolutionised the medical and surgical management of sinonasal disease, and improved understanding of its pathophysiology. As a result, the frequency of external interventions in the frontal and maxillary sinuses was greatly reduced, and the concept of mucosal stripping of the sinuses was largely eliminated as an approach to the resolution of inflammatory disease.

Functional endoscopic sinus surgery provided a number of advantages over traditional, headlightassisted intranasal ethmoidectomy. Although the endoscope did not permit a binocular view and lacked depth perception, it did provide the distinct advantages of a magnified view (allowing detailed examination of the sinuses) and, for the first time, deflected angles of view (allowing surgeons to overcome issues regarding line of sight). Due to its portability, the endoscope proved to be a valuable tool in the office setting, enabling otolaryngologists to perform more thorough examination of the sinonasal cavity. The frequent use of endoscopy, along with the advent of CT, led to improved understanding of

From the Department of Otolaryngology - Head and Neck Surgery, Mount Sinai School of Medicine, New York, New York, and the *Division of Rhinology, Department of Otorhinolaryngology - Head and Neck Surgery, University of Pennsylvania Medical Center, Philadelphia, Pennsylvania, USA.

Accepted for publication: 20 August 2009. First published online 23 November 2009. 
sinonasal anatomy and pathophysiology, and better directed surgical intervention and post-operative care.

Prior to the introduction of endoscopic techniques, despite earlier warnings about the concept from Hilding and others, the primary surgical objective was to remove the 'irreversibly diseased' sinonasal mucosa. Such techniques led to an increase in postoperative scarring and osteoneogenesis, an increase in delayed mucocele formation, and, in certain patients, chronic osteitis and pain. Messerklinger's additional endoscopic insights into pathogenesis and mucociliary clearance reinforced the value of mucosal preservation and led to better directed surgery, focusing initially on the ostiomeatal complex. In Messerklinger's work, significant emphasis was placed on the role of anatomical abnormalities. However, our understanding of the pathogenesis of chronic rhinosinusitis has continued to evolve, and the focus is now on mucosal and bony inflammation. The role of the ostiomeatal complex should not be underestimated as part of the final common pathway creating chronic inflammation; however, it is clear that this is not commonly the underlying cause of sinus disease, and there is now increased recognition of the role of chronic osteitis and the value of 'complete' or total ethmoidectomy, at least in refractory cases.

Over the years, as endoscopic management of inflammatory sinus disease grew more familiar, technological advancements facilitated the development of extended endoscopic procedures, enabling surgery for sinus and skull base tumours, orbital surgery, and closure of skull base defects. This evolution continues with: additional technological innovations; the introduction of minimally invasive balloon techniques for early chronic rhinosinusitis; more reliable methods for closure of large skull base defects; and the potential application of robotic techniques to endoscopic skull base surgery.

\section{History}

Historically, the introduction of nasal endoscopy is largely credited to Hirschmann, who in 1901 attempted an endoscopic examination of the sinonasal cavity using a modified cystoscope. ${ }^{5}$ The following year, Reichert performed what could be regarded as the first endoscopic sinus surgery: some rudimentary maxillary sinus manipulation using a $7-\mathrm{mm}$ endoscope through oroantral fistulae. ${ }^{6}$ In 1925, Maltz promoted the use of endoscopy for diagnostic evaluation of the sinonasal cavity, coining the term 'sinuscopy'. In the 1960s, advancements in optics led to the development of the Hopkins rod system, a major turning point in the field of sinonasal endoscopy.

Professor Harold H Hopkins, who also invented the zoom lens for cameras and the fibre-optic gastroscope, developed the rod optic endoscope system, resulting in enhanced light delivery and superior optical quality. ${ }^{8}$ Using these endoscopes, Walter Messerklinger studied mucociliary clearance in fresh cadavers and subsequently wrote a landmark book on sinonasal endoscopic anatomy and diagnosis, which has shaped our current practice of rhinology. ${ }^{1}$ Wigand, Draf, Messerklinger and others also began performing select surgical procedures, utilising the endoscope and its improved optics. ${ }^{9}$

From the standpoint of the senior author, interest in the potential for endoscopic sinus surgery developed following exposure to: Proctor's teaching regarding the use of endoscopic diagnosis; the frequent failures of Caldwell-Luc surgery; and the apparent success of ethmoidectomy using the operating microscope in the management of chronic rhinosinusitis. The opportunity to review Messerklinger's book on endoscopic diagnosis for a journal, and the subsequent opportunity to meet Messerklinger at a meeting in Dubrovnik, resulted in a letter to Karl Storz about the possibility of obtaining the necessary instrumentation (Figure 1). In subsequent correspondence with Heinz Stammberger, Stammberger stated 'Messerklinger says that this technique means for sinus surgery, what tympanoplasty means for ear surgery'. Rapid growth in endoscopic sinus surgery occurred following two publications delineating the concepts, techniques and imaging parameters for the sinuses. The principles embodied in the concepts of FESS subsequently became the standard of care for chronic inflammatory sinus disease.

\section{Evolution in understanding of pathogenesis}

Prior to the widespread adoption of endoscopic diagnosis and the ability to image the ethmoid sinuses radiographically, Naumann, Drettner, Proctor and others had drawn attention to the importance of the ethmoid sinuses and ostiomeatal complex as key areas in the pathogenesis of chronic inflammatory disease. At this stage, the primary surgical focus in the management of chronic rhinosinusitis remained directed at the maxillary and frontal sinuses, which were more easily visualised on plain radiographs. Accordingly, the most frequent surgical interventions performed for sinusitis were inferior meatal windows or Caldwell-Luc procedures with exenteration of the maxillary sinus mucosa.

As visualisation and the quality of imaging improved, attention became increasingly focused on the anatomical aspects of the ostiomeatal complex and their potential impact on the pathogenesis of chronic rhinosinusitis.

Subsequently, as our understanding of chronic rhinosinusitis has increased, it has become evident how little we know about the underlying factors involved in this disorder. We recognise that the underlying pathology is very rarely anatomical, but rather appears to be a complex activation of the immunological system and a resulting mucosal hyperreactivity. Whereas the underlying aetiology may be multifactorial, with general host, environmental and local predisposing factors, the process appears to be exacerbated by bacterial and fungal contamination and by viral infection (Table I). Our work, and that of others, has clearly shown that the underlying bone becomes involved in this disease process, in a manner similar to its involvement in chronic osteomyelitis. In addition, the inflammation can 


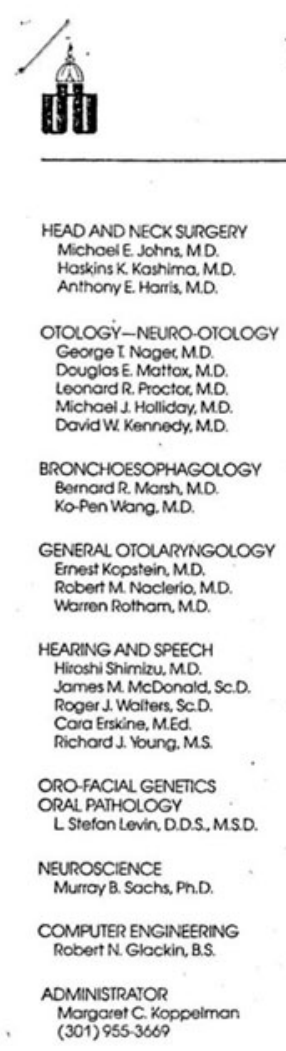

THE JOHNS HOPKINS MEDICAL INSTITUTIONS

Department of Otolaryngology-

Head and Neck Surgery

Norman Silbertrust

Kar1 Storz Endoscopy America Inc.

10111 W. Jefferson Blvd.

Culver City, California 90230

Dear Mr. Silbertrust:

I recently gave some papers at a nasal sinus meeting in Europe, and while there, had the opportunity to listen to and talk with Dr. Walter Messerklinger and one of his associates Dr. Heinz Stamberger. As a result of their presentations and discussions, I became convinced that the techniques of endoscopic sinus surgery which Dr. Messerklinger has advocated and practices, are indeed techniques that will in the future will replace more conventional surgical approaches to sinus disease. I had previously had the opportunity to review $\mathrm{Dr}$. Messerklinger's book on endoscopy of the nose, however, the book did not detail his surgical approaches and in the absence of this, his detailed diagnostic evaluation, described so well in the book, becomes somewhat superfluous and this I believe accounts for the fact that the publication has not been more widely popular in the United States. will probably revolutionize current surgical approaches in the future. Although I am primarily an otologist-neurootologist, I have a significant interest in sinus surgery particularly as

As I mention, I am convinced that endoscopic sinus surgery it relates to both general otolaryngology and to eustachian tube function. As Director of the Resident Training Program at The

FIG. 1

Letter to Mr. Norman Silbertrust of Karl Storz Endoscopy America, Inc.

spread, through the bone, a significant distance away from the primary site of infection. ${ }^{10}$ Although organisms have not been identified within the bone, there appears little doubt that these bony changes are a significant factor in the disease's poor response to medical therapy alone. More recent evidence has examined the role of bacterial superantigens and biofilms as exacerbating factors in this disease process.

Overall, it has become clear that the role of ostiomeatal anatomical variations became dramatically overemphasised by many otolaryngologists in the early post-FESS period. We now recognise that

TABLE I

PREDISPOSING FACTORS FOR CHRONIC RHINOSINUSITIS

Environmental factors

Bacteria, viruses, fungi

Pollution, smoking

Allergens, chemicals

General host factors

Genetic predisposition

Atopy

Immune deficiency

Cystic fibrosis variants, Young's syndrome, etc

Ciliary dyskinesias

Local host factors

Chronic mucosal inflammation

Inflammation in underlying bone

Obstructing neoplasia

Anatomical abnormalities chronic rhinosinusitis is a much more complex, multifactorial problem. Although this was recognised (at least in part) when FESS was first introduced, the message became lost because of the early symptomatic improvement enabled by minimally invasive surgery, and the strong desire for a surgical cure (Figure 2). Indeed, it is possible that some of the observed anatomical abnormalities are actually the result of inflammatory remodelling occurring as part of this complex inflammatory reaction.

\section{Instrumentation}

Following the advent of the Hopkins rod in sinus surgery, the next major evolutionary step was further development of instrumentation. While early endoscopic surgery was often performed with grasping forceps, with limited regard for mucosal preservation, endoscopic follow up demonstrated that denuded bone did not heal well, frequently resulting in scarring, chronic inflammation and occasionally in mucocele formation, just as predicted by Hilding nearly 50 years earlier. Endoscopic follow up helped us see firsthand the sour fruits of our labour. Patients were left with areas of exposed bone and osteoneogenesis. Subsequent remucosalisation in these areas possessed decreased ciliary density and function, occasionally associated with resultant chronic inflammation and pain. ${ }^{11}$ For this reason, we adapted intranasal, fine, through-cutting 


\section{Factors influencing ostiomeatal complex}

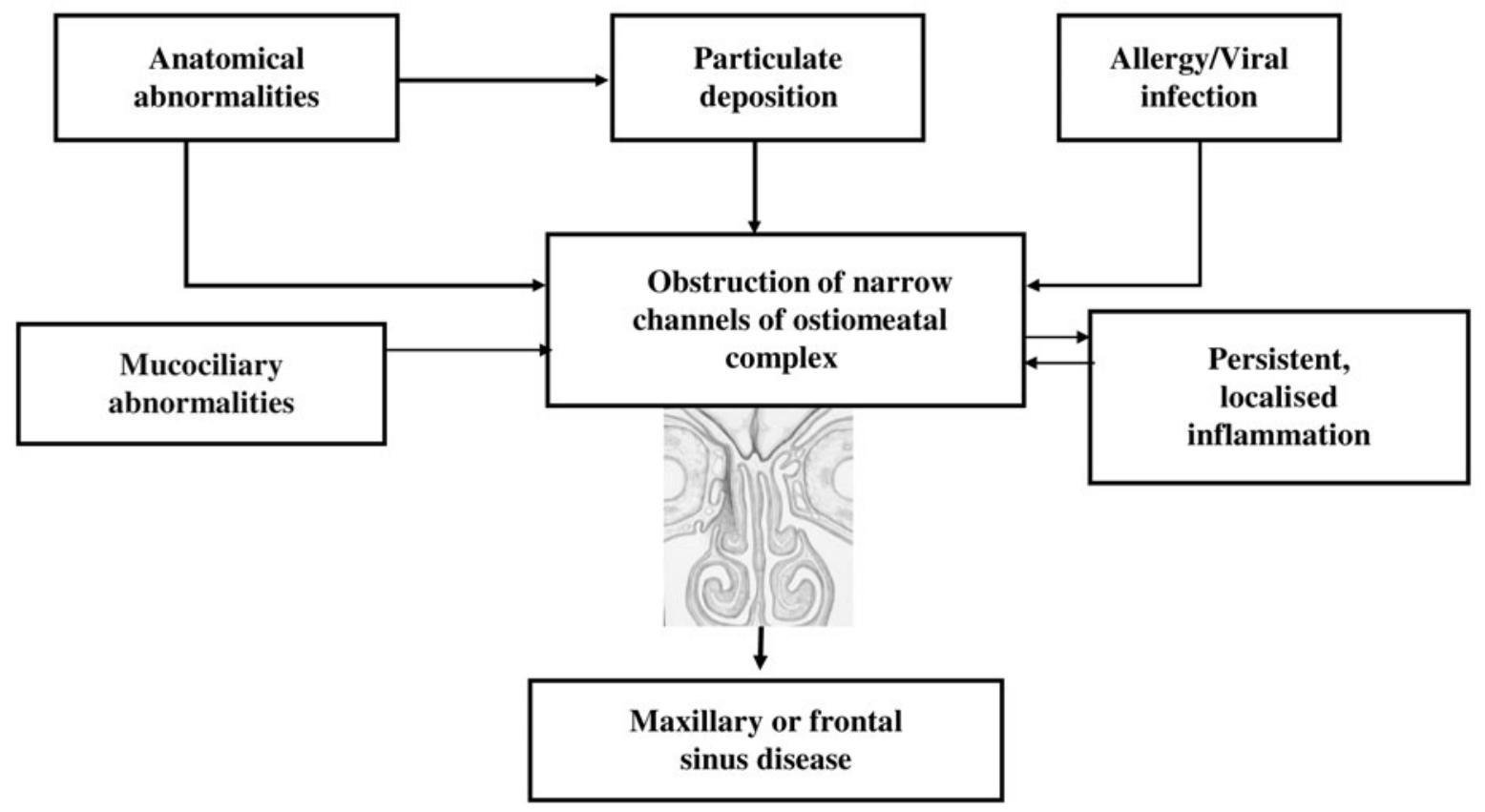

FIG. 2

Teaching diagram (after Draf) used in first endoscopic sinus teaching course. Early in the development of functional endoscopic sinus surgery, the importance of anatomical abnormalities was overemphasised. Despite this, some of the many different factors acting on the ostiomeatal complex were elucidated, and it was recognised that the ostiomeatal complex was really the final common pathway in a complex disease process. Adapted with permission.

instruments originally developed for orthopaedic cartilage removal. Instruments such as straight and $45^{\circ}$ up-biting jaws were initially developed to cut through both bone and mucosa. Although these early instruments were a dramatic improvement on traditional cup forceps, they did occasionally tend to tear mucosa. Ultimately, the development of more precise through-cutting instruments designed specifically for sinonasal use helped alleviate this problem (Figure 3). In no area was the importance of mucosal preservation and reliable through-cutting instrumentation more appreciated than in the frontal recess. This area is bound by stringent anatomical constraints, and fell victim to early attempts at dissection, with subsequent development of osteoneogenesis and delayed mucocele formation. ${ }^{11}$

The development of through-cutting instruments also permitted less traumatic, safer sphenoidotomy. Prior to the development of these instruments, the sphenoid sinus was approached by infracturing through the inferomedial aspect of the last posterior ethmoid cell. This approach had several disadvantages. Entering the sinus by infracturing the anterior wall carried the risk of stripping mucosa adjacent to the natural ostium. Additionally, an infracture sphenoidotomy was a blind procedure, and both intracranial entry and carotid artery rupture were reported as a result, especially in those cases in which an Onodi cell or a shallow sphenoid sinus was present. Through-cutting forceps, on the other hand, allowed the inferior aspect of the superior turbinate to be resected from within the ethmoid sinus, thus providing the surgeon with direct visualisation of the sphenoid ostium while still preserving the precious olfactory fibres of this structure. The natural sphenoid ostium could then be safely enlarged under direct vision, increasing the safety of the procedure.

The microdebrider was a natural evolutionary descendant of through-cutting instrumentation. Originally developed for small joint arthroscopy and cartilage removal in orthopaedic procedures, the device was introduced for endonasal surgery by Setliff and Parsons. ${ }^{12,13}$ The use of disposable blades enabled maintenance of a consistently sharp cutting interface, thus minimising the risk of mucosal stripping. Early microdebriders were slower and obstructed frequently. Subsequent, newer versions were designed to be more efficient and reliably to remove bony partitions, diseased tissue and polyps at a much faster 
(a)

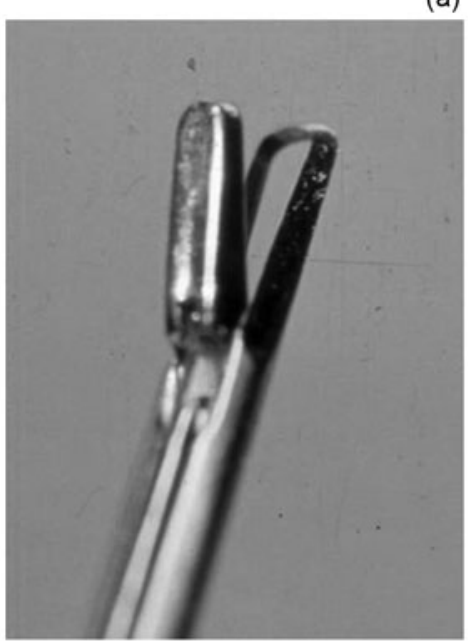

(b)

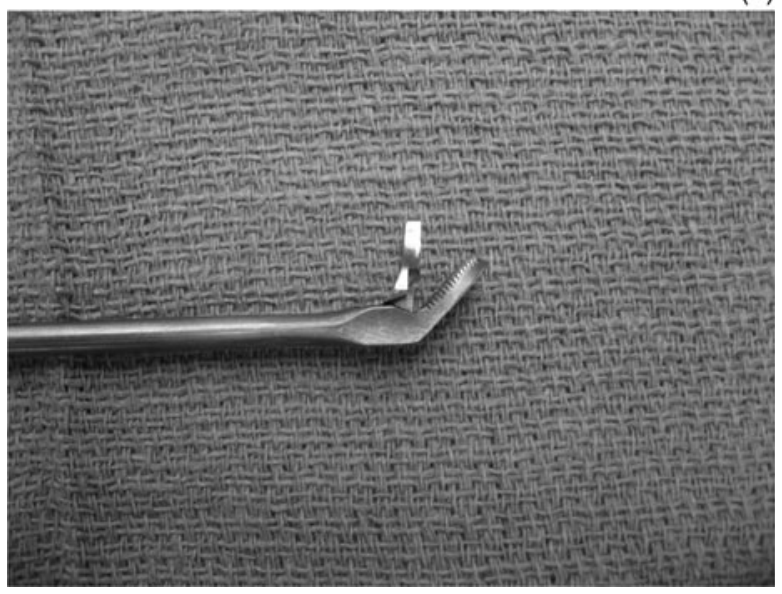

FIG. 3

(a) Early angled, through-cutting instrumentation; early instruments were adapted from the field of orthopaedic surgery. (b) Present straight, through-cutting instrument; instruments were created specifically for sinonasal surgery, with more precise through-cutting capability (Karl-Storz, Tuttlingen, Germany).

rate. The blade designs also continued to evolve, incorporating different sizes and angles, to allow the optimum choice of instrument for each surgical situation. Another major advantage of the microdebrider is its concurrent suction, which enables excellent visualisation even in the presence of significant bleeding. On the other hand, the microdebrider's powered cutting ability, despite its many advantages, may at times prove detrimental. While allowing fast, atraumatic removal of disease, the use of microdebriders resulted in markedly more rapid and severe complications if the surgeon entered the orbit or intracranial cavity. Another disadvantage was the microdebrider's lack of tactile feedback to the surgeon, compared with fine endoscopic instruments. Multiple reports of serious injury to orbital and intracranial contents have been published in the literature. ${ }^{14}$

When using the microdebrider, a suction trap should be used to capture all debrided tissue, with the contents separated by side and submitted for histopathological analysis. This enables compliance with the hospital by-laws of many institutions, and also increases the chance of detecting any occult neoplasm hiding in the surgical specimen. ${ }^{15}$ In addition, when operating the microdebrider, inadvertent trauma can be minimised by ensuring the cutting tip is always visualised, by using the instrument in short bursts, and by keeping it angled away from important structures such as the lamina papyracea. Debrider blades set at an angle of at least $60^{\circ}$ have allowed the removal of redundant mucosa and polyps within the frontal recess, facilitating localisation of the frontal sinus drainage pathway, without mucosal stripping in the hypertrophic chronic sinusitis patient.

Suction-irrigating drills provided another significant advance in instrumentation. Although current suction-irrigation drills are significantly slower than traditional high-speed drills, they provide superior visualisation and safety, and have the advantage of curved shafts. In chronic rhinosinusitis, suction-irrigation drills allow the removal of thickened osteitic bone that cannot be removed with forceps or the microdebrider. While suction-irrigation drills are helpful in some chronic rhinosinusitis surgical procedures, such as extended frontal sinus surgery, they have perhaps more importantly improved our ability to resect intranasal tumours. Not only do they aid in the resection of osseous and fibro-osseous neoplasms, but they also improve our ability to resect soft tissue tumours. We now know it is important to drill or remove the underlying bone in tumours such as inverted papilloma in order to ensure an appropriate tumour resection margin. ${ }^{15}$ Suction-irrigation drills have also been developed which have the appropriate length and angles to access key areas for skull base procedures, such as where the fovea ethmoidalis or planum sphenoidale are traversed to gain access to a tumour of the anterior cranial fossa.

Visualisation has always been an issue in sinus surgery, and key technological improvements in this area have aided our ability to safely operate within the paranasal sinuses. Shapshay and Rebeiz et al. developed a device to clean the tip of the endoscope, termed the 'Endo-Scrub', while experimenting with the use of holmium lasers in endoscopic sinus surgery. ${ }^{16}$ The mechanics of the laser pulse caused blood to splash back onto the lens of the endoscope, necessitating frequent lens cleaning. Shapshay and Rebeiz developed a small syringe attached to a Harvard pump to clean the tip of the endoscope lens during use. This was later modified into the Endo-Scrub pump and Endosheath, which permitted clearance of the endoscope lens within the surgical field. $^{16}$

Traditionally, endoscopes with a 4-mm diameter are used during standard FESS procedures. In paediatric cases, 2.7-mm diameter endoscopes are now available. The standard endoscope length is $18 \mathrm{~cm}$, and has angles of $0^{\circ}, 30^{\circ}, 45^{\circ}$ and $70^{\circ}$. Illumination of the surgical field is achieved via a fibre-optic cable attached to a xenon light source. Light is transmitted along the fibre-optic cable into the Hopkins rod telescope. 
The image quality, however, is dependent on the quality of the camera. Earlier, single chip cameras used a single silicon chip to process all the colours within the surgical field. Three chip cameras were then developed, utilising three silicon chips to represent the main colours red, green and blue. This enhanced the quality of the surgical image, and extended the application of endoscopic surgery to include skull base and intracranial cavity procedures.

With the advent of high definition televisions available to the general consumer, it was inevitable that this level of image quality would extend to the medical field. High definition cameras are now available, and produce images of impeccable quality. The image produced, however, is dependent on the resolution of the monitor displaying the picture. This improvement in image quality arrives at an ideal time, when the scope of the field is expanding exponentially.

Another significant advance in endoscope technology occurred with the introduction of the $45^{\circ}$ wideangle telescope. Providing almost the same deflected angle of view as the $70^{\circ}$ telescope, this telescope is easier to manage because of its forward vision, and it contains a better illumination system.

Several companies have attempted to commercialise three-dimensional endoscopic technology over the years, but, to date, none of their efforts have remained commercially viable. Techniques utilised have included two channel endoscopes, image splitters, electronically generated three-dimensional displays and 'heads up' displays. However, problems with surgeon fatigue, as well as the problem of maintaining the correct orientation of the image when an angled telescope needs to be turned, have, to date, limited the commercial viability of these instruments.

\section{Imaging}

The development of appropriate CT imaging parameters to allow visualisation of the sinuses improved our understanding of chronic rhinosinusitis and provided a major initial impetus to develop the concept of FESS. The subsequent advent of high-resolution CT imaging further facilitated accurate assessment of disease severity, as well as providing anatomical detail to enable pre-operative surgical planning and preparation. The information now available from high-resolution CT scans includes: the presence and extent of skull base erosions; the integrity of the medial orbital wall; the position of the anterior skull base vessels; and the presence of anatomical variants such as Onodi cells. ${ }^{17}$ Magnetic resonance imaging (MRI) also provides useful supplementary sinonasal imaging data. While helpful in differentiating between neoplastic and inflammatory tissue, MRI becomes almost mandatory when sinus opacification abuts an area of skull base erosion, and provides further evaluation and characterisation of skull base malformations including nasal gliomas, meningocoeles and meningoencephalocoeles (Figure 4).

Interactive imaging is a more recent advance in imaging which has improved surgical planning as well as intra-operative decision-making during

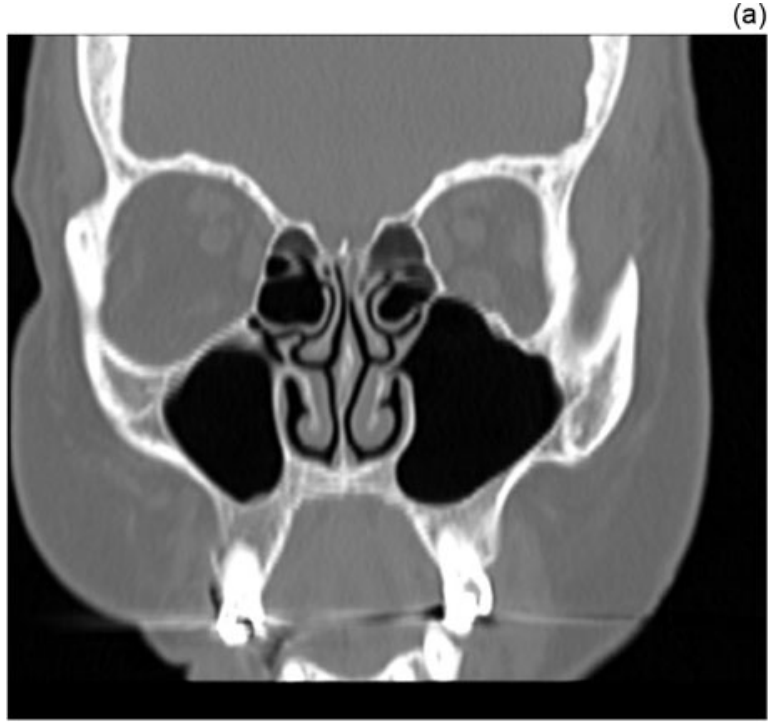

(b)

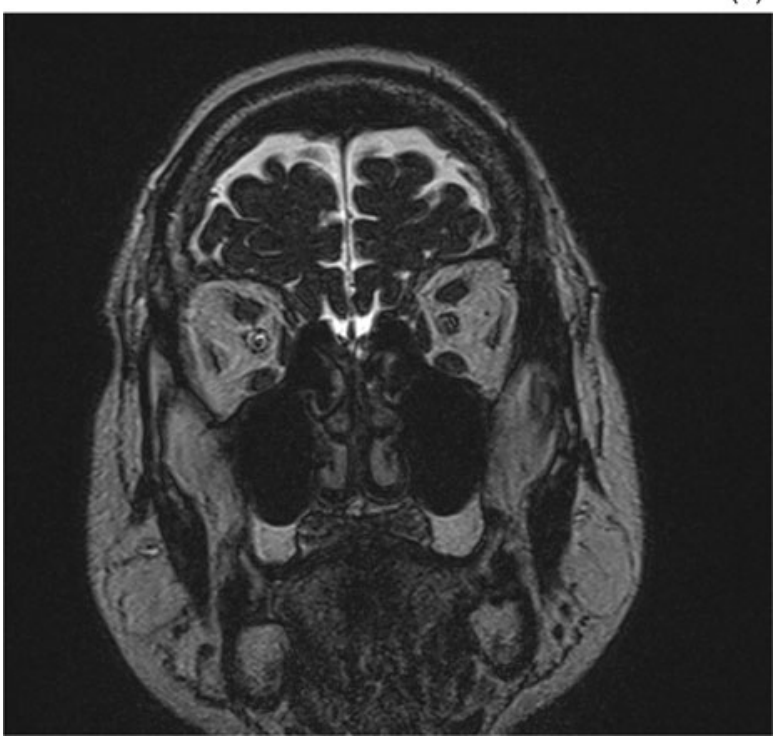

FIG. 4

(a) Coronal, high resolution computed tomography (CT) scan of the paranasal sinuses, showing an area suggestive of skull base dehiscence in the region of the left fovea ethmoidalis. (b) Coronal, high resolution, T2-weighted magnetic resonance imaging (MRI) scan of sinuses, taken to investigate left-sided cerebrospinal fluid (CSF) rhinorrhoea. This MRI was superimposed over the CT (Figure 4a), allowing a pinpoint area of signal intensity identical to CSF to be seen on the left side below the skull base. By combining both imaging modalities, an invasive diagnostic procedure (i.e. CT cisternogram) was avoided in this patient.

FESS. The sinuses provide an ideal scenario for computer image guided surgery based upon preoperative imaging. As long as the surgery is maintained within the rigid bony framework, shifting of soft tissue during the procedure is eliminated, thus allowing the boundaries of the dissection to remain unchanged. The first interactive imaging devices were produced in the late 1980 s by the Aachen group and ISG Technologies, and were based on rigid servo arms and joints. ${ }^{18}$ This system was difficult to set up and manipulate during surgery, and also required head fixation. Early work was also 
performed in the 1980s with electromagnetic devices; however, these devices were extremely inaccurate and easily distorted by minimal magnetic fields (such as those caused by dental fillings). Such inaccuracies were enhanced in an operating theatre containing ferromagnetic instruments and operating room tables. ${ }^{18}$

Today's interactive computer imaging systems have faster registration and touch screens and are less intrusive. There is also a wider range of instrumentation available, which can be tracked during the procedure. However, despite very significant improvements, the current systems are not infallible and do not always provide the desired level of accuracy. The ideal headset has yet to be developed. Movement during the procedure and pressure sore development occasionally occur, and periauricular numbness and facial paralysis resulting from headset usage have been reported.

At this point in time, no strong evidence exists to support the ability of interactive imaging to reduce the incidence of complications. The reported mean accuracy of image guidance systems is $2 \mathrm{~mm}$ or less. ${ }^{19}$ Nevertheless, computer interactive imaging is clearly helpful with regard to orientation during extensive and revision surgical procedures, frontal

(a)

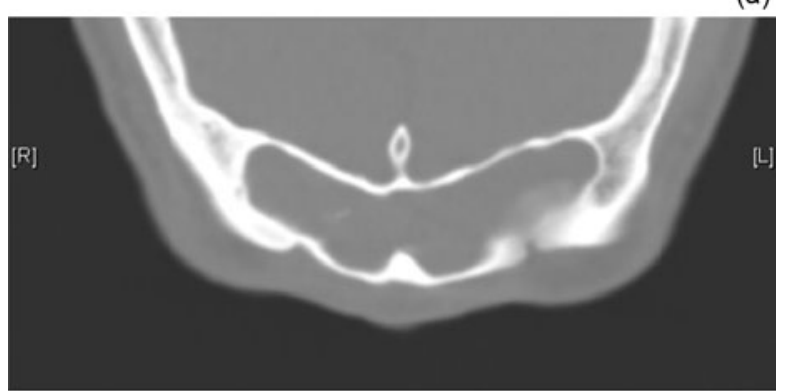

(b)

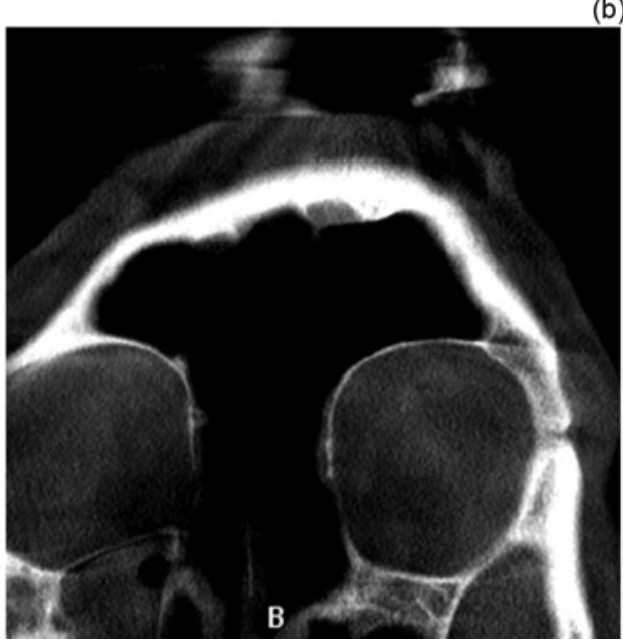

FIG. 5

(a) Pre-operative, axial computed tomography (CT) scan showing a frontal sinus inverted papilloma. (b) Intra-operative, coronal CT scan taken after endoscopic tumour resection via a Draf 3 approach to the frontal sinus. Note that the tumour has been completely removed, leaving a post-operative cavity amenable to tumour surveillance. $\mathrm{R}=$ right; $\mathrm{L}=$ left. recess dissection, and, particularly, fibro-osseous tumour removal. There is also evidence to suggest that it may aid the completeness of surgical procedures. However, perhaps one of the most significant benefits of computer interactive imaging is the ability to scroll through images in three planes repeatedly, which in turn allows the surgeon to conceptualise the three-dimensional anatomy of this complex area, and thus formulate a surgical plan prior to the procedure. ${ }^{20}$

Intra-operative CT and/or MRI imaging is currently being utilised at a limited number of institutions (Figure 5). Intra-operative CT using volumetric CT scanners is relatively inexpensive and has a very low irradiation dose; in the future, it is anticipated that this could directly provide intra-operative computerassisted navigation. Even in the case of inflammatory disease, intra-operative CT scanning has demonstrated an incidence of residual bony partitions of more than 30 per cent at the conclusion of a surgical procedure. ${ }^{21}$ Currently, the data have to be manually uploaded to the computer-assisted navigation system when required. Intra-operative MRI is rarely used at many institutions and has a limited role, because of logistical reasons including the amount of space needed in the operating theatre, the preparation time required, and the extremely high cost of the equipment and nonferromagnetic instrumentation.

\section{Balloon sinuplasty}

Balloon sinuplasty deserves mentioning as a technology which continues to evolve. It is unclear at this point what ultimate impact this technology will have upon endoscopic sinus surgery. Balloon sinuplasty was prompted by the success of catheter technology in other medical disciplines, including cardiology, urology, gastroenterology and vascular surgery. The sinus ostium is entered in an atraumatic fashion via a catheter-based system, and the ostia is dilated while minimising injury to surrounding tissue. Balloon sinuplasty was initially developed in 2002 by engineers based in California, USA. After development of patents and further instrumentation, the company Acclarent (Menlo Park, California, USA) was formed. ${ }^{22}$

The first human trials of balloon sinuplasty were originally performed in Australia. The first multicentre trial was entitled 'Safety and outcomes of balloon catheter sinusotomy: a multicenter 24 -week analysis in 115 patients'. 22 This was a prospective, nonrandomised trial conducted by 10 experienced rhinologists. The data demonstrated a high patency rate for maxillary and frontal sinuses at six months, and a high indeterminate rate for the sphenoid sinus (39 per cent). ${ }^{22}$

Further, long-term studies will be necessary before final recommendations on balloon sinuplasty can be made. Currently, the indications for balloon sinuplasty are unclear, and its use is limited since it is not recommended in cases of polypoid disease and it does not address disease in the most commonly involved sinus, the ethmoid sinus. However, when 
the procedure is combined with drug-eluting stents or with other future innovations, its potential may be significant. Currently, a spacer balloon is available for the frontal sinus which can elute steroid over a two week period, and additional, bioabsorbable stents are being tested in clinical trials. Thus, in the future, it may be possible to apply minimally invasive treatments and to diminish the role of surgery in certain conditions. $^{23}$

\section{Tumour, orbital and skull base surgery}

Shortly following the introduction of endoscopic sinus surgery, it became evident that certain benign tumours could be removed under endoscopic visualisation. By the late 1980s, surgeons had begun to perform endoscopically some skull base tumour resections, orbital decompressions, malignant tumour resections and pituitary procedures. The first series of endoscopic pituitary surgery procedures was published by Jankowski in $1992 .{ }^{24}$ Since that time, there has been an exponential growth in endoscopic skull base surgery, with better understanding of the principles involved in endoscopic oncological surgery and more reliable skull base reconstruction.

At this point in time, the general principles of endoscopic tumour removal are meticulous identification of the site (or sites) of tumour origin, by either debulking or working around the tumour, and meticulous haemostasis. In the case of benign tumours such as inverted papilloma, the underlying bone is then either burred down or removed at the site of tumour attachment. In the case of malignant tumour, a true oncological resection is performed, with resection of a significant margin of normal tissue and bone, so that the resection is never compromised by performing the surgery endoscopically. Where skull base resection is necessary, wide removal of bone and cauterisation of the appropriate ethmoidal arteries precedes dural resection. Although the ability to close wide-field dural resections has improved with the use of arterially based vascular flaps such as the Haddad flap, endoscopic intracranial tumour removal is still limited by our ability to satisfactorily control intracranial bleeding. ${ }^{25}$ An ideal suction bipolar cautery has yet to be developed, and there is room for further improvement in skull base instrumentation.

Although robot systems have now been very satisfactorily utilised for trans-oral laryngeal and hypopharyngeal surgery, the instrumentation remains too large for practical trans-nasal skull base procedures. When smaller, more appropriate robot instrumentation is developed, the potential for skull base and trans-nasal intracranial surgery will be significantly enhanced; hopefully, such technology will enable the avoidance of a significant number of open procedures for intracranial skull base lesions, along with the associated brain retraction and secondary morbidity.

\section{Conclusion}

Technical innovations in the field of endoscopic sinus surgery have helped transform the management of patients with sinonasal disorders. In just over two decades, we have seen the field progress from open surgical procedures frequently focused on mucosal stripping, with headlight-assisted, intranasal ethmoidectomy being the standard of care, to functional endoscopic procedures utilising high definition cameras and stereotactic surgical navigation, with the facility for intra-operative CT. Improvements in optics and instrumentation have spawned the birth of a new field, endoscopic skull base surgery, which spares patients the morbidity of a craniotomy and the cerebral oedema associated with frontal lobe retraction. The boundaries of our specialty continue to evolve rapidly. Where we will be in several decades' time has yet to be determined, but the future shows great promise.

\section{References}

1 Messerklinger W. Endoscopy of the Nose. Baltimore, Maryland: Urban \& Schwarzenberg, 1978

2 Kennedy DW. Functional endoscopic sinus surgery. Technique. Arch Otolaryngol 1985;111:643-9

3 Kennedy DW, Zinreich SJ, Rosenbaum AE, Johns ME. Functional endoscopic sinus surgery. Theory and diagnostic evaluation. Arch Otolaryngol 1985;111:576-82

4 Schaefer SD. An anatomic approach to endoscopic intranasal ethmoidectomy. Laryngoscope 1998;108:1628-34

5 Jacobs JB. 100 years of frontal sinus surgery. Laryngoscope 1997; 107:1-36

6 Pownell PH, Minoli JJ, Rohrich RJ. Diagnostic nasal endoscopy. Plast Reconstr Surg 1997;99:1451-8

7 Cohen NA, Kennedy DW. Endoscopic sinus surgery: where we are - and where we're going. Curr Opin Otolaryngol Head Neck Surg 2005;13:32-8

8 Jennings CR. Harold Hopkins. Arch Otolaryngol Head Neck Surg 1998;124:1042

9 Hosemann W. Surgical treatment of nasal polyposis in patients with aspirin intolerance. Thorax 2000;55(Suppl. 2):S87-90

10 Perloff JR, Gannon FH, Bolger WE, Montone KT, Orlandi $\mathrm{R}$, Kennedy DW. Bone involvement in sinusitis: an apparent pathway for the spread of disease. Laryngoscope 2000; 110:2095-9

11 Kennedy DW. Functional Endoscopic Sinus Surgery: Concepts, Surgical Indications, and Instrumentation. In: Kennedy DW, BolgerWE: Zubreich SJ. Hamilton, Diseases of the Sinuses Diagnosis and Management, Ontario: BC Decker, 2000;197-210

12 Setliff RC 3rd. The hummer: a remedy for apprehension in functional endoscopic sinus surgery. Otolaryngol Clin North Am 1996;29:95-104

13 Parsons DS. Rhinologic uses of powered instrumentation in children beyond sinus surgery. Otolaryngol Clin North Am 1996;29:105-14

14 Hackman TG, Ferguson BJ. Powered instrumentation and tissue effects in the nose and paranasal sinuses. Curr Opin Otolaryngol Head Neck Surg 2005;13:22-6

15 Kennedy DW. Technical innovations and the evolution of endoscopic sinus surgery. Ann Otol Rhinol Laryngol Suppl 2006;196:3-12

16 Shapshay SM, Rebeiz EE, Pankratov MM. Holmium: yttrium aluminum garnet laser-assisted endoscopic sinus surgery: clinical experience. Laryngoscope 1992;102: $1177-80$

17 Stamm AM. Transnasal endoscopy-assisted skull base surgery. Ann Otol Rhinol Laryngol Suppl 2006;196:45-53

18 Anon JB, Klimek L, Mosges R, Zinreich SJ. Computerassisted endoscopic sinus surgery. An international review. Otolaryngol Clin North Am 1997;30:389-401

19 Labadie RF, Davis BM, Fitzpatrick JM. Image-guided surgery: what is the accuracy? Curr Opin Otolaryngol Head Neck Surg 2005;13:27-31

20 Moharir VM, Fried MP, Vernick DM, Janecka IP, Zahajsky J, Hsu L et al. Computer-assisted three- 
dimensional reconstruction of head and neck tumors. Laryngoscope 1998;108:1592-8

21 Jackman AH, Palmer JN, Chiu AG, Kennedy DW. Use of intraoperative CT scanning in endoscopic sinus surgery: a preliminary report. Am J Rhinol 2008;22:170-4

22 Bolger WE, Brown CL, Church CA, Goldberg AN, Karanfilov B, Kuhn FA et al. Safety and outcomes of balloon catheter sinusotomy: a multicenter 24-week analysis in 115 patients. Otolaryngol Head Neck Surg 2007; 137:10-20

23 Lanza DC, Kennedy DW. Balloon sinuplasty: not ready for prime time. Ann Otol Rhinol Laryngol 2006;115:789-90, $91-2$

24 Jankowski R, Auque J, Simon C, Marchal JC, Hepner H, Wayoff M. Endoscopic pituitary tumor surgery. Laryngoscope 1992;102:198-202

25 Hadad G, Bassagasteguy L, Carrau RL, Mataza JC, Kassam A, Snyderman $\mathrm{CH}$ et al. A novel reconstructive technique after endoscopic expanded endonasal approaches: vascular pedicle nasoseptal flap. Laryngoscope 2006;116:1882-6
Address for correspondence:

Prof David W Kennedy,

Division of Rhinology,

Department of Otorhinolaryngology - Head and

Neck Surgery,

Hospital of the University of Pennsylvania

5 Silverstein/Ravdin,

3400 Spruce St, Philadelphia,

PA 19104, USA.

E-mail: David.Kennedy@uphs.upenn.edu

Professor D W Kennedy was one of The Journal of Laryngology \& Otology Visiting Professors, 2009.

Professor D W Kennedy takes responsibility for the integrity of the content of the paper. Competing interests: None declared 